\title{
FIXED POINT FORMULA FOR CHARACTERS OF AUTOMORPHISM GROUPS ASSOCIATED WITH KÄHLER CLASSES
}

\author{
Akito Futaki and Kenji Tsuboi
}

\section{Introduction}

The existence problem of Kähler-Einstein metrics of positive scalar curvature has not been settled yet completely. There are known obstructions, among which there is a character $f$ of the complex Lie algebra $\mathfrak{h}(M)$ of all holomorphic vector fields defined in [8]. In our works [11], [10], [13], [21], we studied how this Lie algebra character lifted to a character of the group of biholomorphic automomorphisms. It was shown using Chern-Simons theory that it is lifted to an additive group character with values in $\mathbb{C} / \mathbb{Z}$. The imaginary part was written as an integral formula, while the $\mathbb{R} / \mathbb{Z}$-valued real part was given as a fixed point formula for automorphisms.

On the other hand the Lie algebra character $f$ can be extended to an obstruction $f_{\Omega}$ for Kähler classes $\Omega$ to contain a constant scalar curvature metric [9], [6]. More precisely, let $M$ be an $m$-dimensional compact Kähler manifold with a fixed Kähler class $\Omega, \omega \in \Omega$ a Kähler form and $s_{\omega}$ the scalar curvature of $\omega$. Then there exists a smooth function $F_{\omega}$ uniquely up to constants such that

$$
s_{\omega}-m \mu_{\Omega}=\Delta F_{\omega}
$$

where

$$
\mu_{\Omega}=\frac{\left(\Omega^{m-1} \cup c_{1}(M)\right)[M]}{\Omega^{m}[M]} .
$$

If we define $f_{\Omega}: \mathfrak{h}(M) \rightarrow \mathbb{C}$ by

$$
f_{\Omega}(X)=\frac{1}{2 \pi} \int_{M} X F_{\omega} \omega^{m}
$$

then the right hand side is independent of the choice of Kähler forms $\omega \in \Omega$ and therefore invariant under the group of $\Omega$-preserving automorphisms of $M$. This last fact implies that $f_{\Omega}$ is a Lie algebra character (c.f. [6]). (Bando [3] further extended $f_{\Omega}$ as obstructions for Kähler classes to contain Kähler metrics with harmonic Chern forms of higher degree ; note that Kähler metrics with constant scalar curvature are exactly those with harmonic first Chern forms.)

In a more recent work of Nakagawa [18] it is shown that, when the Kähler class $\Omega$ is a Hodge class and a holomorphic line bundle $L$ with $c_{1}(L)=\Omega$ admits a

Received May 10, 2001. 
lifting of the action of a subgroup $G$ in the group of $\Omega$-preserving automorphisms of $M$, the Lie algebra character $f_{\Omega}$ lifts to a group character $\hat{f}_{\Omega}$ of $G$ with values in $\mathbb{C} /\left(\mathbb{Z}+\mu_{\Omega} \mathbb{Z}\right)$. In $[12]$ the imaginary part of the group character $\hat{f}_{\Omega}$ is given as an integral formula. As a byproduct we see, when $\Omega=c_{1}(M)$, Mabuchi's K-energy functional [16] and a functional introduced by Ding [5] are included in a family of functionals with cocycle conditions. The purpose of the present paper is to compute the real part of the group character $\hat{f}_{\Omega}$ by expressing it in terms of the fixed point set of the automorphisms. This is done by interpreting the real part as an eta invariant of a Dirac operator on a mapping torus and applying results of Atiyah-Patodi-Singer [1], Atiyah-Singer [2], Donnelly [7].

\section{Fixed point formula}

To begin with we briefly review basic facts about characteristic classes of foliations. A transeversely holomorphic foliation $\mathcal{F}$ of complex codimension $m$ on a smooth manifold $B$ of real dimension $2 m+n$ is given by a system of local charts $\left\{z^{1} \cdots, z^{m}, x^{1}, \cdots, x^{n}\right\}$ where $\left\{x^{1}, \cdots, x^{n}\right\}$ are real coordinates along the leaves and $\left\{z^{1}, \cdots, z^{m}\right\}$ are complex coordinates in the normal directions, such that for any neighboring local charts $\left\{w^{1} \cdots, w^{m}, y^{1}, \cdots, y^{n}\right\}$, the $w^{i}$ 's are holomorphic functions of only $z^{i}$ 's. Then there is a subbundle $T_{1,0}^{*}$ of $T B^{*} \otimes \mathbb{C}$ spanned by $\left\{d z^{1}, \cdots, d z^{m}\right\}$ in local charts. Note that the definition of $T_{1,0}^{*}$ is independent of the choice of local charts. A section of $T_{1,0}^{*}$ will be called a differential form of type $(1,0)$. Let $\mathcal{E} \rightarrow B$ be a complex vector bundle of rank $r$ over $B$. A basic connection or Bott connection of $\mathcal{E}$ is a linear connection whose connection form is of type $(1,0)$. It is obvious from the dimension reasons that, for a multi-index $\alpha$ with $|\alpha|>m$, the Chern form $c^{\alpha}(\mathcal{E}, \nabla)$ vanishes identically if $\nabla$ is a basic connection. The Chern-Simons theory tells us that then we have a Simons class $S_{c_{1}^{m+1}}(\mathcal{E}) \in H^{2 m+1}(B ; \mathbb{C} / \mathbb{Z})$ which is independent of the choice of basic connections.

Let $M$ be an $m$-dimensional connected compact complex manifold, Aut $(M)$ the complex Lie group consisting of all biholomorphic automorphisms of $M$. Consider a holomorphic vector bundle $E \rightarrow M$ to which the action of a subgroup $G \subset \operatorname{Aut}(M)$ lifts. Choose an automophism $\sigma \in G$. We set $E_{\sigma}:=(\mathbb{R} \times E) / \mathbb{Z}$ and $M_{\sigma}:=(\mathbb{R} \times M) / \mathbb{Z}$, where $\mathbb{Z}$ acts on $\mathbb{R} \times E$ by

$$
n \cdot(t, v)=\left(t-n, \sigma^{n}(v)\right), \quad n \in \mathbb{Z}
$$

and on $\mathbb{R} \times M$ similarly. There is a natural transversely holomorphic foliation on $\mathbb{R} \times M$ with leaf dimension 1 , and it descends to $M_{\sigma}$. It is easy to see that the complex vector bundle $E_{\sigma} \rightarrow M_{\sigma}$ carries basic connections (c.f. [12]).

Hence we can define $\hat{f}_{E}: G \rightarrow \mathbb{C} / \mathbb{Z}$ by

$$
\hat{f}_{E}(\sigma)=S_{c_{1}^{m+1}}\left(E_{\sigma}\right)\left[M_{\sigma}\right]
$$

where $\left[M_{\sigma}\right]$ is the fundamental cycle of $\left[M_{\sigma}\right]$. One can see that $\hat{f}_{E}(\sigma)$ defines an additive group character, i.e. $\hat{f}_{E}(\sigma \tau)=\hat{f}_{E}(\sigma)+\hat{f}_{E}(\tau)$ (c.f.[11], [18]). 
Suppose, for our purposes, that the subgroup $G$ of Aut $(M)$ preserves a Hodge class $\Omega \in H^{1,1}(M ; \mathbb{Z})$ and also that the $G$-action lifts to an action on a holomorphic line bundle $L$ with $c_{1}(L)=\Omega$. On the other hand, there exist natural $G$-actions on the canonical bundle $K_{M}^{+1}:=K_{M}$ and the anticanonical bundle $K_{M}^{-1}$. Let $\mu_{\Omega}$ be the rational number defined in the Introduction.

Thorem 2.1 (c.f. [18]). Define a group homomorphism $\hat{f}_{\Omega}: G \longrightarrow \mathbb{C} /\left(\mathbb{Z}+\mu_{\Omega} \mathbb{Z}\right)$ by

$$
\begin{aligned}
\hat{f}_{\Omega}(\sigma):= & (m+1) \sum_{i=0}^{m}(-1)^{i}\left(\begin{array}{c}
m \\
i
\end{array}\right) S_{c_{1}^{m+1}}\left(\left(K_{M}^{-1} \otimes L^{m-2 i}\right)_{\sigma}\right)\left[M_{\sigma}\right] \\
& -(m+1) \sum_{i=0}^{m}(-1)^{i}\left(\begin{array}{c}
m \\
i
\end{array}\right) S_{c_{1}^{m+1}}\left(\left(K_{M} \otimes L^{m-2 i}\right)_{\sigma}\right)\left[M_{\sigma}\right] \\
& -m \mu_{\Omega} \sum_{i=0}^{m+1}(-1)^{i}\left(\begin{array}{c}
m+1 \\
i
\end{array}\right) S_{c_{1}^{m+1}}\left(\left(L^{m+1-2 i}\right)_{\sigma}\right)\left[M_{\sigma}\right]
\end{aligned}
$$

Then this character $\hat{f}_{\Omega}$ is a lift of the Lie algebra character $f_{\Omega}$ associated with the given Kähler class $\Omega$ mentioned in the Introduction.

We outline the proof of this theorem for the reader's convenience. First we take the derivative of $\hat{f}_{\Omega}\left(\sigma_{t}\right)$ with respect to $t$ where $\sigma_{t}$ is a flow generated by a holomorphic vector field. If we use (6.3) in [20], we can see that good cancelations occur and that the derivative coincides with the Lie algebra character which obstructs the existence of constant curvature metric in $\Omega$.

Now we wish to write $\hat{f}_{\Omega}(\sigma)$ in terms of fixed point set of $\sigma$. From now on we assume that $\sigma \in \operatorname{Aut}(M)$ is a periodic element of order $p \geq 2$ and let $G$ be the cyclic subgroup of Aut $(M)$ generated by $\sigma$. Put $U:=D^{2} \times M$ and $Y:=S^{1} \times M$, and denote by $q_{D}: U \longrightarrow D^{2}$ and $q_{S}: Y \longrightarrow S^{1}$ the first factor projections, and by $q_{U}: U \longrightarrow M$ and $q_{Y}: Y \longrightarrow M$ the second factor projections. Then the cyclic group $G$ acts on $U$ and $Y$ as follows:

$$
\sigma \cdot\left(r e^{i \theta}, z\right)=\left(r e^{i(\theta-2 \pi / p)}, \sigma \cdot z\right)
$$

for $\left(r e^{i \theta}, z\right) \in U=D^{2} \times M$. Then the $G$-action on $Y$ is free and $M_{\sigma}$ is identified with $Y / G$. Note that the fixed point set of the $\sigma^{k}$-action on $U$ is contained in $M=\{0\} \times M \subset U$ and coincides with that of the $\sigma^{k}$-action on $M$.

Assumption 2.2. (a) There exists a compact $2 m+2$-dimensional almost complex manifold $W$ with boundary $M_{\sigma}$ which is isomorphic to $U / G$ near the boundary as an almost complex manifold.

(b) The line bundle $L_{\sigma}=\left(q_{Y}^{*} L\right) / G$ extends to a smooth complex line bundle $L_{W}$ on $W$ above.

Note that, since the direct sum of the tangent bundle of $M_{\sigma}$ and the trivial real line bundle has a complex structure, it follows from the result of [17] that the condition (a) is always satisfied, but the authors do not know if the condition 
(b) is always satisfied. Note also that then $K_{W}^{+1}:=K_{W}=\wedge^{m+1} T^{*} W$ gives an extension of $\left(q_{Y}^{*} K_{M}\right) / G$ and $K_{W}^{-1}=\wedge^{m+1} T W$ gives an extension of $\left(q_{Y}^{*} K_{M}^{-1}\right) / G$.

Assumption 2.2 is satisfied by a wide class of examples as the following lemma shows.

Lemma 2.3. Suppose that the fixed point set of $\sigma^{k}$-action on $M$ is independent of $k$ and that every connected component of the fixed point set has a cell decomposition with no codimension one cells. Then Assumption 2.2 is satisfied.

Proof. The singularities of $U / G$ are cyclic quotient singularities in the normal directions along the fixed point set of $G$. Let $N_{j} \subset M=\{0\} \times M \subset U$ be a connected component of the fixed point set and $D_{j}$ the disk bundle of the normal bundle of $N_{j}$ in $U$ with sufficiently small radius with respect to a $G$-invariant metric such that $D_{j}$ 's are mutually disjoint. Note that each $N_{j}$ is a $G$-invariant retract of $D_{j}$. Let $\Sigma$ be the union of $D_{j}$, which is a $G$-invariant subset of $U$. Then $G$ acts freely on $U-\Sigma$ and hence $\left(\left.q_{U}^{*} L\right|_{U-\Sigma}\right) / G$ gives an extension of $L_{\sigma}$ on $(U-\Sigma) / G$. On the other hand, $\Sigma / G$ is the disjoint union of the neighborhoods $V_{i}$ of the singular points. Let $\widetilde{V}_{i}$ be a resolution of $V_{i}$ for each $i$ which obviously gives a resolution $W$ of $U / G$. Then the $G$-invariant retractions of $D_{j}$ give retractions of $\widetilde{V}_{i}$ to the exceptional divisors $E_{i}$. To show that $L_{\sigma}$ extends over $W$ we have only to show that every smooth complex line bundle over $\partial \widetilde{V}_{i}$ extends to $\widetilde{V}_{i}$. First note that the group of isomorphism classes of smooth complex line bundles is isomorphic to the 2-dimensional integral cohomology group. From the exact sequence

$$
\cdots \longrightarrow H^{2}\left(\widetilde{V}_{i} ; \mathbb{Z}\right) \longrightarrow H^{2}\left(\partial \widetilde{V}_{i} ; \mathbb{Z}\right) \longrightarrow H^{3}\left(\widetilde{V}_{i}, \partial \widetilde{V}_{i} ; \mathbb{Z}\right) \longrightarrow \cdots
$$

we have only to show that $H^{3}\left(\widetilde{V}_{i}, \partial \widetilde{V}_{i} ; \mathbb{Z}\right)=0$. But

$$
H^{3}\left(\widetilde{V}_{i}, \partial \widetilde{V}_{i} ; \mathbb{Z}\right) \cong H_{2 m-1}\left(\widetilde{V}_{i} ; \mathbb{Z}\right) \cong H_{2 m-1}\left(E_{i} ; \mathbb{Z}\right) .
$$

Hence it suffices to show that each $E_{i}$ has a cell decomposition with no codimension one cells. $E_{i}$ is a fibration over the fixed point set with fiber isomorphic to the exceptional divisor of the resolution for an isolated cyclic quotient singularity, and thus we need only to see that the exceptional divisor for an isolated cyclic quotient singularity has no codimension one cells. We embed our situation in a quotient of $n$-dimensional complex projective space $\mathbb{P}^{n}$ as follows. Let $H$ be a hyperplane. Then $\mathbb{P}^{n}-H \cong \mathbb{C}^{n}$ and we may assume the $G$ is a subgroup of the torus $\left(\mathbb{C}^{*}\right)^{n}$. Then the singularity is embedded as the origin in $\mathbb{C}^{n} / G$. This cyclic quotient singularity is a toric singularity and can be resolved equivariantly, see e.g. Oda [19]. Let $\widetilde{\mathbb{P}}$ be the equivariant resolution. Then we apply a theorem of Bialynicki-Birula [4] (see also Kirwan [14] for a moment map proof) to $\widetilde{\mathbb{P}}$ and see that the exceptional divisor, which is an invariant subvariety, can be stratified by strata isomorphic to the total spaces of vector bundles over components of the fixed point set. Since the fixed point set is a union of complex submanifolds of complex codimension at least one, it follows that the exceptional divisor has no codimension one cells. This completes the proof of the lemma. 
Definition 2.4. Let $S(k)$ be the fixed point set of $\sigma^{k}$ consisting of compact connected complex submanifolds $N$ of $M$ and $\nu(N, M)$ the normal bundle of $N$ in $M$. Then $\nu(N, M)$ is decomposed into the direct sum of subbundles

$$
\nu(N, M)=\oplus_{j} \nu\left(N, \theta_{j}\right)
$$

where $\sigma^{k}$ acts on $\nu\left(N, \theta_{j}\right)$ via multiplication by $e^{i \theta_{j}}$. We define the characteristic class $\Phi(\nu(N, M))$ by

$$
\Phi(\nu(N, M))=\prod_{j} \prod_{k=1}^{r_{j}} \frac{1}{1-e^{-x_{k}-i \theta_{j}}} \in H^{*}(N ; \mathbb{C}) \quad\left(r_{j}=\operatorname{rank}_{\mathbb{C}}\left(\nu\left(N, \theta_{j}\right)\right)\right)
$$

where $\prod_{k}\left(1+x_{k}\right)$ is equal to the total Chern class of $\nu\left(N, \theta_{j}\right)$.

Thorem 2.5. Suppose that $G$ is a cyclic group generated by an automorphism $\sigma$ of order $p$ and that Assumption 2.2 is satisfied. Then the character $\hat{f}_{\Omega}(\sigma)$ can be written in terms of the fixed point set of $\sigma^{k}$ as follows. Let $\alpha$ be the primitive $p$-th root of unity and assume that $\sigma^{k}$ acts on $\left.K_{M}\right|_{N}$ via multiplication by $\alpha^{\beta}$ and acts on $\left.L\right|_{N}$ via multiplication by $\alpha^{\gamma}$ for $\beta, \gamma \in \mathbb{Z}$. Then for any integer $n$ we have

$$
\begin{aligned}
& S_{c_{1}^{m+1}}\left(\left(K_{M}^{ \pm 1} \otimes L^{n}\right)_{\sigma}\right)\left[M_{\sigma}\right]= \\
& \frac{1}{p} \sum_{k=1}^{p-1} \sum_{N \subset S(k)} \frac{1}{1-\alpha^{k}}\left(\alpha^{ \pm \beta+n \gamma} e^{c_{1}\left(K_{M}^{ \pm 1} \mid N\right)+n(\Omega \mid N)}-1\right)^{m+1} \operatorname{Td}(T N) \Phi(\nu(N, M))[N] \\
& S_{c_{1}^{m+1}\left(\left(L^{n}\right)_{\sigma}\right)\left[M_{\sigma}\right]=} \\
& \quad \frac{1}{p} \sum_{k=1}^{p-1} \sum_{N \subset S(k)} \frac{1}{1-\alpha^{k}}\left(\alpha^{n \gamma} e^{n(\Omega \mid N)}-1\right)^{m+1} \operatorname{Td}(T N) \Phi(\nu(N, M))[N]
\end{aligned}
$$

where $\operatorname{Td}(T N)$ is the Todd class of $T N$ and $[N]$ is the fundamental cycle of $N$.

Proof. We regard $U:=D^{2} \times M, Y:=S^{1} \times M$ as the $\operatorname{spin}^{c}$-manifolds with the $\operatorname{spin}^{c}$-structures defined by the $\mathrm{U}(m)$-structure of $M$ and the trivial $\operatorname{spin}^{c}$ structures of $D^{2}, S^{1}$ respectively. If we choose $G$-invariant Hermitian metrics $h$ and $h^{L}$ of $T M$ and $L$, then we have the unique $G$-invariant Hermitian connections, i.e. type $(1,0)$ metric connections, $\nabla$ and $\nabla^{L}$ of $T M$ and $L$ respectively. We also give a rotationally symmetric Hermitian metric $h^{D}$ on the complex manifold $D^{2}$ such that it is a product metric of $S^{1} \times[0, \delta)$ near $\partial D^{2}=S^{1}$. Let $\nabla^{D}$ be the $G$-invariant Hermitian connection of $T D^{2}$. Then the $G$-invariant Hermitian metric $h^{U}$ on $U$ is defined by $h$ and $h^{D}$. Let $\nabla^{U}$ be the $G$-invariant $h^{U}$-preserving type $(1,0)$ connection of $T U$, which is the direct sum connection of $\nabla$ and $\nabla^{D}$. Now let $E^{ \pm}$be virtual complex line bundles with $G$-invariant metric connections defined by

$$
E^{ \pm}=\otimes^{m+1}\left(K_{M}^{ \pm 1} \otimes L^{n}-\varepsilon\right)
$$


where $\varepsilon$ is the trivial complex line bundle with the trivial connection and the trivial $G$-action. Then using the spin ${ }^{c}$-structures, the metrics and the connections of $E^{ \pm}, T U$ and $T Y$, we can define the $\operatorname{spin}^{c}$-Dirac operators (or Dolbeault operators)

$D_{U}: \Gamma\left(S_{U}^{+} \otimes q_{U}^{*} E^{ \pm}\right) \longrightarrow \Gamma\left(S_{U}^{-} \otimes q_{U}^{*} E^{ \pm}\right), D_{Y}: \Gamma\left(S_{Y} \otimes q_{Y}^{*} E^{ \pm}\right) \longrightarrow \Gamma\left(S_{Y} \otimes q_{Y}^{*} E^{ \pm}\right)$

where $S_{U}^{ \pm}$is the half spinor bundles over $U$ and $S_{Y}=\left.S_{U}^{+}\right|_{Y}=\left.S_{U}^{-}\right|_{Y}$ is the spinor bundle over $Y$. Then the $G$-equivariant operator $D_{Y}$ naturally defines a self-adjoint elliptic operator $D_{\sigma}$ on $M_{\sigma}$, which is the $q_{Y}^{*} E^{ \pm} / G$-valued $\operatorname{spin}^{c}$ Dirac operator on $M_{\sigma}$. Let $\eta\left(D_{\sigma}\right)$ be the eta invariant of $D_{\sigma}, \eta\left(D_{Y}, \sigma^{k}\right)$ the eta invariant of $D_{Y}$ evaluated at $\sigma^{k}$ and set

$\xi\left(D_{\sigma}\right):=\frac{\eta\left(D_{\sigma}\right)+\operatorname{dim} \operatorname{ker}\left(D_{\sigma}\right)}{2}, \quad \xi\left(D_{Y}, \sigma^{k}\right):=\frac{\eta\left(D_{Y}, \sigma^{k}\right)+\operatorname{Tr}\left(\sigma^{k} \mid \operatorname{ker}\left(D_{Y}\right)\right)}{2}$.

Then since $D_{U}$ is expressed as

$$
D_{U}=\tau\left(\frac{\partial}{\partial u}+D_{Y}\right)
$$

on the collar $Y \times[0, \delta) \subset U$ where $u$ is the coordinate of $[0, \delta)$ and $\tau$ is a bundle isomorphism, it follows from Theorem(3.10) in [1], Theorem 1.2 in [7], Theo$\operatorname{rem}(4.3),(4.6)$ in $[2]$ that

(1) $\xi\left(D_{Y}\right)=\xi\left(D_{Y}, 1\right)=\int_{U} \operatorname{Ch}\left(q_{U}^{*} E^{ \pm}\right) \operatorname{Td}(T U)-\operatorname{Index}\left(D_{U}, 1\right)$

$$
\xi\left(D_{Y}, \sigma^{k}\right)=\sum_{N \subset S(k)} \operatorname{Ch}\left(\left.E^{ \pm}\right|_{N}, \sigma^{k}\right) \operatorname{Td}(T N) \Phi(\nu(N, U))[N]-\operatorname{Index}\left(D_{U}, \sigma^{k}\right)
$$

for $1 \leq k \leq p-1$ where $\operatorname{Ch}\left(q_{U}^{*} E^{ \pm}\right)$is the Chern character form of $q_{U}^{*} E^{ \pm}, \operatorname{Td}(T U)$ is the Todd form of $T U, \mathrm{Ch}\left(\left.E^{ \pm}\right|_{N}, \sigma^{k}\right)$ is the Chern character of $\left.E^{ \pm}\right|_{N}$ evaluated at $\sigma^{k}$ and $\operatorname{Index}\left(D_{U}, \sigma^{k}\right)$ is the index of $D_{U}$ with a certain global boundary condition evaluated at $\sigma^{k}$. Then since the connection of $q_{U}^{*} E^{ \pm}$is induced from that of $E^{ \pm}$, it follows that

$\operatorname{Ch}\left(q_{U}^{*} E^{ \pm}\right)=q_{U}^{*} \operatorname{Ch}\left(\otimes^{m+1}\left(K_{M}^{ \pm 1} \otimes L^{n}-\varepsilon\right)\right)=q_{U}^{*}\left(\exp \left(c_{1}\left(K_{M}^{ \pm 1} \otimes L^{n}\right)\right)-1\right)^{m+1}=0$

from the dimension reasons and hence it follows from (1) that

$$
\xi\left(D_{Y}, 1\right)=-\operatorname{Index}\left(D_{U}, 1\right) .
$$

Since $\quad \sum_{k=1}^{p} \operatorname{Tr}\left(\left.\sigma^{k}\right|_{V}\right) \equiv 0(\bmod p) \quad$ for any complex $G$-module $V$, we have

$$
\sum_{k=1}^{p} \operatorname{Index}\left(D_{U}, \sigma^{k}\right) \equiv 0 \quad(\bmod p)
$$


and therefore it follows from (2), (3) that

$$
\begin{aligned}
\sum_{k=1}^{p} \xi\left(D_{Y}, \sigma^{k}\right) \equiv & \\
& \sum_{k=1}^{p-1} \sum_{N \subset S(k)} \operatorname{Ch}\left(\left.E^{ \pm}\right|_{N}, \sigma^{k}\right) \operatorname{Td}(T N) \Phi(\nu(N, U))[N] \quad(\bmod p) .
\end{aligned}
$$

Set $U_{0}:=\left(D^{2}-\{0\}\right) \times M$ and let $q_{U_{0}}: U_{0} \longrightarrow M$ be the second factor projection. Then the connections $\nabla$ and $\nabla^{U}$ naturally define connections $q_{Y}^{*} \nabla / G$ of $T M_{\sigma}$ and $\nabla^{U} / G$ of $T\left(U_{0} / G\right)$. Moreover the connection $\nabla^{L}$ defines connections $q_{Y}^{*} \nabla^{L} / G$ of $L_{\sigma}$ and $q_{U_{0}}^{*} \nabla^{L} / G$ of $q_{U_{0}}^{*} L / G$. Note that the connections $q_{Y}^{*} \nabla / G, q_{Y}^{*} \nabla^{L} / G$ define a type $(1,0)$ connections of the line bundles $\left(K_{M}^{ \pm 1} \otimes L^{n}\right)_{\sigma}=q_{Y}^{*}\left(K_{M}^{ \pm 1} \otimes L^{n}\right) / G$ for any integer $n$. The connection $q_{Y}^{*} \nabla / G$ extends to a metric connection of $T W$ which coincides with $\nabla^{U} / G$ near $M_{\sigma}$, and the connection $q_{Y}^{*} \nabla^{L} / G$ extends to a metric connection of $L_{W}$ which coincides with $q_{U_{0}}^{*} \nabla^{L} / G$ near $M_{\sigma}$. The connection of $T W$ defines metric connections of $K_{W}^{ \pm 1}$ and the half spinor bundles $S_{W}^{ \pm}$over $W$ with respect to the natural $\operatorname{spin}^{c}$-structure of $W$. Let

$$
D_{W}: \Gamma\left(S_{W}^{+} \otimes\left(\otimes^{m+1}\left(K_{W}^{ \pm 1} \otimes L_{W}^{n}-\varepsilon\right)\right)\right) \longrightarrow \Gamma\left(S_{W}^{-} \otimes\left(\otimes^{m+1}\left(K_{W}^{ \pm 1} \otimes L_{W}^{n}-\varepsilon\right)\right)\right)
$$

be the $\otimes^{m+1}\left(K_{W}^{ \pm 1} \otimes L_{W}^{n}-\varepsilon\right)$-valued $\operatorname{spin}^{c}$-Dirac operator on $W$ defined by using the connections defined above. Then as in (1) we have

$$
\xi\left(D_{\sigma}\right)=\int_{W} \operatorname{Ch}\left(\otimes^{m+1}\left(K_{W}^{ \pm 1} \otimes L_{W}^{n}-\varepsilon\right)\right) \operatorname{Td}(T W)-\operatorname{Index}\left(D_{W}, 1\right) .
$$

Now the Dirac operators $D_{\sigma}, D_{Y}$ split into $D_{\sigma}=D_{\sigma}^{+} \oplus\left(D_{\sigma}^{+}\right)^{*}, D_{Y}=D_{Y}^{+} \oplus$ $\left(D_{Y}^{+}\right)^{*}$ because the $\operatorname{spin}^{c}(2 m+1)$-structures of $M_{\sigma}$ and $Y$ come from the $\mathrm{U}(m)$ structure of $M$. Since the dimensions of $M_{\sigma}$ and $Y$ are odd and $\sigma^{k}(1 \leq k \leq p-1)$ acts freely on $Y$, it follows from the result in [2] that

$$
\begin{aligned}
& \operatorname{Index}\left(D_{\sigma}^{+}\right)=\operatorname{dim} \operatorname{ker}\left(D_{\sigma}^{+}\right)-\operatorname{dim} \operatorname{ker}\left(\left(D_{\sigma}^{+}\right)^{*}\right)=0, \\
& \operatorname{Index}\left(D_{Y}, \sigma^{k}\right)=\operatorname{Tr}\left(\sigma^{k} \mid \operatorname{ker}\left(D_{Y}^{+}\right)\right)-\operatorname{Tr}\left(\sigma^{k} \mid \operatorname{ker}\left(\left(D_{Y}^{+}\right)^{*}\right)\right)=0
\end{aligned}
$$

for any $k$. Therefore it follows that

$$
\begin{aligned}
& \frac{1}{2} \operatorname{dim} \operatorname{ker}\left(D_{\sigma}\right)=\operatorname{dim} \operatorname{ker}\left(D_{\sigma}^{+}\right) \equiv 0 \quad(\bmod \mathbb{Z}), \\
& \frac{1}{p} \sum_{k=1}^{p} \frac{1}{2} \operatorname{Tr}\left(\sigma^{k} \mid \operatorname{ker}\left(D_{Y}\right)\right)=\frac{1}{p} \sum_{k=1}^{p} \operatorname{Tr}\left(\sigma^{k} \mid \operatorname{ker}\left(D_{Y}^{+}\right)\right) \equiv 0 \quad(\bmod \mathbb{Z}) .
\end{aligned}
$$

Hence it follows from (3.6) in [7] that

$$
\xi\left(D_{\sigma}\right) \equiv \frac{1}{2} \eta\left(D_{\sigma}\right)=\frac{1}{p} \sum_{k=1}^{p} \frac{1}{2} \eta\left(D_{Y}, \sigma^{k}\right) \equiv \frac{1}{p} \sum_{k=1}^{p} \xi\left(D_{Y}, \sigma^{k}\right) \quad(\bmod \mathbb{Z}),
$$


and therefore it follows from (4), (5) that

$$
\begin{aligned}
& S_{c_{1}^{m+1}}\left(\left(K_{M}^{ \pm 1} \otimes L^{n}\right)_{\sigma}\right)\left[M_{\sigma}\right] \\
& =S_{c_{1}^{m+1}}\left(q_{Y}^{*}\left(K_{M}^{ \pm 1} \otimes L^{n}\right) / G\right)\left[M_{\sigma}\right]=S_{c_{1}^{m+1}}\left(\left.\left(K_{W}^{ \pm 1} \otimes L_{W}^{n}\right)\right|_{\partial W}\right)[\partial W] \\
& \equiv \int_{W} c_{1}\left(K_{W}^{ \pm 1} \otimes L_{W}^{n}\right)^{m+1}=\int_{W} \operatorname{Ch}\left(\otimes^{m+1}\left(K_{W}^{ \pm 1} \otimes L_{W}^{n}-\varepsilon\right)\right) \operatorname{Td}(T W) \\
& \equiv \xi\left(D_{\sigma}\right) \equiv \frac{1}{p} \sum_{k=1}^{p} \xi\left(D_{Y}, \sigma^{k}\right) \\
& \equiv \frac{1}{p} \sum_{k=1}^{p-1} \sum_{N \subset S(k)} \operatorname{Ch}\left(\left.E^{ \pm}\right|_{N}, \sigma^{k}\right) \operatorname{Td}(T N) \Phi(\nu(N, U))[N] \\
& =\frac{1}{p} \sum_{k=1}^{p-1} \sum_{N \subset S(k)} \\
& \left(\operatorname{Ch}\left(\left(\left.K_{M}^{ \pm 1}\right|_{N}\right) \otimes\left(\left.L\right|_{N}\right)^{n}, \sigma^{k}\right)-1\right)^{m+1} \operatorname{Td}(T N) \frac{1}{1-\alpha^{k}} \Phi(\nu(N, M))[N] \\
& =\frac{1}{p} \sum_{k=1}^{p-1} \sum_{N \subset S(k)} \\
& \frac{1}{1-\alpha^{k}}\left(\alpha^{ \pm \beta+n \gamma} e^{c_{1}\left(\left.K_{M}^{ \pm 1}\right|_{N}\right)+n\left(\left.\Omega\right|_{N}\right)}-1\right)^{m+1} \operatorname{Td}(T N) \Phi(\nu(N, M))[N]
\end{aligned}
$$

where $\equiv$ denotes the equivalence $\bmod \mathbb{Z}$. The remaining equality is proved similarly. This completes the proof of Theorem 2.5.

Remark 2.6. Let $D: \Gamma\left(S^{+} \otimes E^{ \pm}\right) \longrightarrow \Gamma\left(S^{-} \otimes E^{ \pm}\right)$be the $\operatorname{spin}^{c}$-Dirac operator on $M$, which is a $G$-equivariant operator. Then since

$$
\operatorname{Index}(D)=\mathrm{Ch}\left(E^{ \pm}\right) \operatorname{Td}(T M)[M]=c_{1}\left(K_{M}^{ \pm 1} \otimes L^{n}\right)^{m+1} \operatorname{Td}(T M)[M]=0,
$$

it follows from the same argument as in [22] that

$$
\begin{gathered}
\operatorname{det}\left(\left.\sigma\right|_{\operatorname{ker}(D)}\right) / \operatorname{det}\left(\left.\sigma\right|_{\operatorname{ker}\left(D^{*}\right)}\right)=\exp \frac{2 \pi i}{p} \sum_{k=1}^{p-1} \frac{1}{1-\alpha^{k}} \operatorname{Index}\left(D, \sigma^{k}\right) \\
=\exp \frac{2 \pi i}{p} \sum_{k=1}^{p-1} \sum_{N \subset S(k)} \\
\frac{1}{1-\alpha^{k}}\left(\alpha^{ \pm \beta+n \gamma} e^{c_{1}\left(\left.K_{M}^{ \pm 1}\right|_{N}\right)+n\left(\left.\Omega\right|_{N}\right)}-1\right)^{m+1} \operatorname{Td}(T N) \Phi(\nu(N, M))[N] .
\end{gathered}
$$

Hence we can see that

$$
\begin{gathered}
\sigma \rightarrow \frac{1}{p} \sum_{k=1}^{p-1} \sum_{N \subset S(k)} \\
\frac{1}{1-\alpha^{k}}\left(\alpha^{ \pm \beta+n \gamma} e^{c_{1}\left(\left.K_{M}^{ \pm 1}\right|_{N}\right)+n\left(\left.\Omega\right|_{N}\right)}-1\right)^{m+1} \operatorname{Td}(T N) \Phi(\nu(N, M))[N]
\end{gathered}
$$


defines an additive group character $G \rightarrow \mathbb{C} / \mathbb{Z}$.

\section{Example}

In this section we compute an example. Notice that our example below satisfies Assumption 2.2 by virtue of Lemma 2.3. Let $M:=\mathbb{C P}^{2}$ be the 2-dimensional complex projective space and $\Omega$ the positive generator of $H^{2}(M ; \mathbb{Z}) \cong \mathbb{Z}$. Then $\Omega=c_{1}(L)$ where $L$ is the hyperplane bundle over $M$. For any natural number $p \geq 2$, an automorphism $\sigma$ of $M$ is defined by

$$
\sigma:\left[z_{0}: z_{1}: z_{2}\right] \longrightarrow\left[\alpha z_{0}: z_{1}: z_{2}\right]
$$

where $\alpha$ is the primitive $p$-th root of unity. This action has a finite order $p$ and lifts to an action on $L$. Since $\sigma=\exp X$ for the holomorphic vector field $X$ on $M$ represented by the diagonal matrix with diagonal entries $2 \pi i / p, 0,0$ and $\Omega$ contains a Kähler metric with constant scalar curvature, it follows from Theorem 2.1 that $\hat{f}_{\Omega}(\sigma)$ must vanish. This is also verified by using Theorem 2.5 as follows.

It is clear that the fixed point set $N$ of $\sigma^{k}$-action is independent of $k$ and coincides with the disjoint union of the point $q=[1: 0: 0]$ and the hyperplane $H=\left\{z_{0}=0\right\}$, which have cell decomposions with no codimension one cells. Then $\sigma^{k}$ acts on the normal bundle $\nu(q, M)$ via multiplication by $\alpha^{-k}$ and acts

on the normal bundle $\nu(H, M)=L \mid H$ via multiplication by $\alpha^{k}$. Moreover we have

$$
\begin{aligned}
& \sigma^{k}\left|\left(K_{M}^{-1} \mid q\right)=\alpha^{-2 k}, \quad \sigma^{k}\right|\left(K_{M}^{-1} \mid H\right)=\alpha^{k}, \quad \sigma^{k} \mid(L \mid q)=\alpha^{-k}, \\
& \sigma^{k} \mid(L \mid H)=\alpha^{k}, \quad c_{1}\left(K_{M}^{-1} \mid q\right)=c_{1}(L \mid q)=0, \quad c_{1}\left(K_{M}^{-1} \mid H\right)=c_{1}(T M \mid H)=3 x
\end{aligned}
$$

where $x:=c_{1}(L \mid H)$ is the positive generator of $H^{2}(H ; \mathbb{Z})=H^{2}\left(\mathbb{C P}^{1} ; \mathbb{Z}\right)$. Set

$$
\psi(u)=u^{s}\left(\frac{1-u^{t}}{1-u}\right)^{\ell}
$$

for any integer $s, t$ and any natural number $\ell$. Then since $\psi(1)=t^{\ell}$, we have

$$
\frac{1}{p} \sum_{k=1}^{p-1} \alpha^{s k}\left(\frac{1-\alpha^{t k}}{1-\alpha^{k}}\right)^{\ell} \equiv \frac{1}{p} \sum_{k=1}^{p-1} \alpha^{s k}\left(\frac{1-\alpha^{-t k}}{1-\alpha^{-k}}\right)^{\ell} \equiv \frac{-t^{\ell}}{p} \quad(\bmod \mathbb{Z})
$$

because $\quad \sum_{k=1}^{p-1} \alpha^{r k} \equiv-1(\bmod p) \quad$ for any integer $r$. 
Now, for any integer $n$, the following equalities follows from Theorem 2.5.

$$
\begin{aligned}
& S_{c_{1}^{3}}\left(\left(K_{M}^{-1} \otimes L^{n}\right)_{\sigma}\right)\left[M_{\sigma}\right] \\
& =\frac{1}{p} \sum_{k=1}^{p-1} \frac{1}{1-\alpha^{k}}\left(\alpha^{k+n k} e^{3 x+n x}-1\right)^{3}(1+x) \frac{1}{1-\alpha^{-k} e^{-x}}[H] \\
& +\frac{1}{p} \sum_{k=1}^{p-1} \frac{1}{1-\alpha^{k}}\left(\alpha^{-2 k-n k}-1\right)^{3}\left(\frac{1}{1-\alpha^{k}}\right)^{2}[q] \\
& =\frac{1}{p} \sum_{k=1}^{p-1}\left(\left(\alpha^{(n+1) k}-1\right)^{3}+3(n+3) \alpha^{(n+1) k}\left(\alpha^{(n+1) k}-1\right)^{2} x\right) \\
& (1+x)\left(-\frac{\alpha^{k}}{\left(\alpha^{k}-1\right)^{2}}+\frac{\alpha^{k}}{\left(\alpha^{k}-1\right)^{3}} x\right)[H] \\
& +\frac{1}{p} \sum_{k=1}^{p-1} \alpha^{-3 k}\left(\frac{1-\alpha^{-(n+2) k}}{1-\alpha^{-k}}\right)^{3} \\
& =-\frac{1}{p} \sum_{k=1}^{p-1} \alpha^{k}\left(\alpha^{(n+1) k}-1\right)\left(\frac{1-\alpha^{(n+1) k}}{1-\alpha^{k}}\right)^{2}+\frac{1}{p} \sum_{k=1}^{p-1} \alpha^{k}\left(\frac{1-\alpha^{(n+1) k}}{1-\alpha^{k}}\right)^{3} \\
& -\frac{1}{p} \sum_{k=1}^{p-1} 3(n+3) \alpha^{(n+2) k}\left(\frac{1-\alpha^{(n+1) k}}{1-\alpha^{k}}\right)^{2} \\
& +\frac{1}{p} \sum_{k=1}^{p-1} \alpha^{-3 k}\left(\frac{1-\alpha^{-(n+2) k}}{1-\alpha^{-k}}\right)^{3} \\
& \equiv \frac{1}{p}\left\{(1-1)(n+1)^{2}-(n+1)^{3}+3(n+3)(n+1)^{2}-(n+2)^{3}\right\} \\
& =\frac{1}{p}\left(n^{3}+6 n^{2}+6 n\right) \quad(\bmod \mathbb{Z}) .
\end{aligned}
$$

Now since $K_{M} \cong\left(K_{M}^{-1}\right)^{*}$, a similar calculation shows that

$$
\begin{aligned}
& S_{C_{1}^{3}}\left(\left(K_{M} \otimes L^{n}\right)_{\sigma}\right)\left[M_{\sigma}\right] \\
& =\frac{1}{p} \sum_{k=1}^{p-1} \frac{1}{1-\alpha^{k}}\left(\alpha^{-k+n k} e^{-3 x+n x}-1\right)^{3}(1+x) \frac{1}{1-\alpha^{-k} e^{-x}}[H] \\
& \quad+\frac{1}{p} \sum_{k=1}^{p-1} \frac{1}{1-\alpha^{k}}\left(\alpha^{2 k-n k}-1\right)^{3}\left(\frac{1}{1-\alpha^{k}}\right)^{2}[q] \\
& =\frac{1}{p}\left(n^{3}-6 n^{2}+6 n\right) \quad(\bmod \mathbb{Z}),
\end{aligned}
$$




$$
\begin{aligned}
& S_{c_{1}^{3}}\left(\left(L^{n}\right)_{\sigma}\right)\left[M_{\sigma}\right] \\
& =\frac{1}{p} \sum_{k=1}^{p-1} \frac{1}{1-\alpha^{k}}\left(\alpha^{n k} e^{n x}-1\right)^{3}(1+x) \frac{1}{1-\alpha^{-k} e^{-x}}[H] \\
& \quad+\frac{1}{p} \sum_{k=1}^{p-1} \frac{1}{1-\alpha^{k}}\left(\alpha^{-n k}-\right)^{3}\left(\frac{1}{1-\alpha^{k}}\right)^{2}[q] \\
& =\frac{1}{p} n^{3} \quad(\bmod \mathbb{Z}) .
\end{aligned}
$$

Hence we have

$$
\begin{aligned}
& \sum_{i=0}^{2}(-1)^{i}\left(\begin{array}{c}
2 \\
i
\end{array}\right)\left\{S_{c_{1}^{3}}\left(\left(K_{M}^{-1} \otimes L^{2-2 i}\right)_{\sigma}\right)\left[M_{\sigma}\right]-S_{c_{1}^{3}}\left(\left(K_{M} \otimes L^{2-2 i}\right)_{\sigma}\right)\left[M_{\sigma}\right]\right\} \\
& =\frac{1}{p} \sum_{i=0}^{2}(-1)^{i}\left(\begin{array}{c}
2 \\
i
\end{array}\right) 12(2-2 i)^{2}=96 / p \\
& \sum_{i=0}^{3}(-1)^{i}\left(\begin{array}{c}
3 \\
i
\end{array}\right)\left\{S_{c_{1}^{3}}\left(\left(L^{3-2 i}\right)_{\sigma}\right)\left[M_{\sigma}\right]\right\} \\
& =\frac{1}{p} \sum_{i=0}^{3}(-1)^{i}\left(\begin{array}{c}
3 \\
i
\end{array}\right)(3-2 i)^{3}=48 / p
\end{aligned}
$$

Now since $\Omega^{2}=c_{1}(L)^{2}$ is the positive generator of $H^{4}(M ; \mathbb{Z})$, we have

$$
\mu_{\Omega}=\frac{\Omega^{2-1} \cup c_{1}(M)[M]}{\Omega^{2}[M]}=\frac{\Omega^{2-1} \cup(3 \Omega)[M]}{\Omega^{2}[M]}=3 .
$$

Hence we have

$$
\begin{aligned}
\hat{f}_{\Omega}(\sigma):= & 3 \sum_{i=0}^{2}(-1)^{i}\left(\begin{array}{c}
2 \\
i
\end{array}\right) \\
\left\{S_{c_{1}^{3}}\left(\left(K_{M}^{-1} \otimes L^{2-2 i}\right)_{\sigma}\right)\left[M_{\sigma}\right]-S_{c_{1}^{3}}\left(\left(K_{M} \otimes L^{2-2 i}\right)_{\sigma}\right)\left[M_{\sigma}\right]\right\} & \\
& -2 \cdot 3 \sum_{i=0}^{3}(-1)^{i}\left(\begin{array}{c}
3 \\
i
\end{array}\right) S_{c_{1}^{3}}\left(\left(L^{3-2 i}\right)_{\sigma}\right)\left[M_{\sigma}\right] \\
= & \{3 \cdot 96-2 \cdot 3 \cdot 48\} / p=0 .
\end{aligned}
$$

One can find many examples of computations for the Lie algebra character $f_{\Omega}$ in [15] and could try similar computations for our group character $\hat{f}_{\Omega}$.

\section{Acknowledgement:}

We have benefited from conversations with Sadayoshi Kojima. 


\section{References}

[1] M. F. Atiyah, V. K. Patodi and I. M. Singer. Spectral asymmetry and Riemannian geometry I, Math. Proc. Camb. Phil. Soc. 77, 43-69 (1975).

[2] M. F. Atiyah and I. M. Singer. The index of elliptic operators III, Ann. of Math. 87, 546-604 (1968).

[3] S. Bando. An obstruction for Chern class forms to be harmonic, preprint (1983).

[4] A. Bialynicki-Birula. Some theorems on actions of algebraic groups, Ann. of Math. 98, 480-497(1973).

[5] W. Ding. Remarks on the existence problem of positive Kähler-Einstein metrics, Math. Ann. 282, 463-471 (1988).

[6] E. Calabi. Extremal Kähler metrics II, Differential geometry and complex analysis, (I. Chavel and H.M. Farkas eds.), 95-114, Springer-Verlag, Berline-Heidelberg-New York, (1985).

[7] H. Donnelly. Eta invariants for G-spaces, Indiana Math. J. 27, 889-918 (1978).

[8] A. Futaki. An obstruction to the existence of Einstein Kähler metrics, Invent. Math. 73, 437-443 (1983).

[9] A. Futaki. On compact Kähler manifold of constant scalar curvature, Proc. Japan Acad., Ser. A, 59, 401-402 (1983).

[10] A. Futaki. On a character of the automorphism group of a compact complex manifold, Invent. Math. 87, 655-660 (1987).

[11] A. Futaki and S. Morita. Invariant polynomials of the automophism group of a compact complex manifold, J. Diff. Geom. 21, 135-142 (1985).

[12] A. Futaki and Y. Nakagawa. Characters of automorphism groups associated with Kähler classes and functionals with cocycle conditions, to appear in Kodai Math. J.

[13] A. Futaki and K. Tsuboi. Eta invariants and automorphisms of compact complex manifolds, Recent Topics in Differential and Analytic Geometry, Adv. Stud. Pure Math. 18-I, 251-270 (1990), Kinokuniya Tokyo.

[14] F. C. Kirwan. Cohomology of quotients in symplectic and algebraic geometry, Princeton University Press, New Jersey, (1984).

[15] C. LeBrun and S. R. Simanca. Extremal Kähler metrics and complex deformation theory, Geometry and Global Analysis, 107-153, Tohoku University, Sendai, Japan (1993)

[16] T. Mabuchi. K-energy maps integrating Futaki invariants, Tohoku Math. J., 38, 245-257 (1987).

[17] S. Morita. Almost complex manifolds and Hirzebruch invariant for isolated singularities, Math. Ann. 211, 245-260(1974).

[18] Y. Nakagawa. The Bando-Calabi-Futaki character and its lifting to a group character, preprint.

[19] T. Oda. Convex bodies and algebraic geometry-An introduction to the theory of toric varieties, Ergeb. Math. Grenzgeb. 15, Springer-Verlag, Berlin-Heidelberg, (1988).

[20] G. Tian. Kähler-Einstein metrics on algebraic manifolds, Proc. C.I.M.E. conference on Transcendetal methods in algebraic geometry, Lecture Notes in Math., vol. 1646, 143-185, Springer-Verlag, Berline-Heidelberg-New York, (1996).

[21] K. Tsuboi. The lifted Futaki invariants and the Spin $^{c}$-Dirac operators, Osaka J. Math. 32, 207-225 (1995).

[22] K. Tsuboi. On the determinant and the holonomy of equivariant elliptic operators, Proc. A.M.S. 123, 2275-2281 (1995). 
Department of Mathematics, Tokyo Institute of Technology, 2-12-1 Oh-OKayama, Meguro, Tokyo 152-8551, JAPAN

E-mail address: futaki@math.titech.ac.jp

Department of Mathematics, Tokyo University of Fisheries, 4-5-7 Kohnan, Minato, TOKYO 108-8477, JAPAN

E-mail address: tsuboi@tokyo-u-fish.ac.jp. 